\title{
Development of rabbit monoclonal and polyclonal antibodies for detection of site-specific histone modifications and their application in analyzing overall modification levels
}

\author{
Lan Guo ${ }^{1,2, *}$, Benliang Yin ${ }^{1, *}$, Junli Zhou ${ }^{1,3}$, Xueyong Li $^{3}$, Xing Wang Deng ${ }^{1,2,3}$ \\ ${ }^{I}$ National Institute of Biological Science, Zhongguancun Life Science Park, Beijing 102206, China; ${ }^{2}$ Peking-Yale Joint Center of \\ Plant Molecular Genetics and Agrobiotechnology, College of Life Sciences, Peking University, Beijing 100871, China; ${ }^{3}$ Department \\ of Molecular, Cellular, and Developmental Biology, Yale University, New Haven, Connecticut 06520-8104, USA
}

In addition to DNA sequence information, site-specific histone modifications are another important determinant of gene expression in a eukaryotic organism. We selected four modification sites in common histones that are known to significantly impact chromatin function and generated monoclonal or polyclonal antibodies that recognize each of those site-specific modifications. We used these antibodies to demonstrate that the site-specific histone modification levels remain relatively constant in different organs of the same organism. We also compared the levels of selected histone modifications among several representative organisms and found that site-specific modifications are highly variable among different organisms, providing new insight into the evolutionary divergence of specific histone modifications.

Cell Research (2006) 16:519-527. doi:10.1038/sj.cr.7310063; published online 15 May 2006

Keywords: histone modification, site-specific antibody, chromatin

\section{Introduction}

Histone modification encompasses a diverse array of post-translational covalent modifications that are found primarily on the histone N-terminus [1]. Many sites in histones are subject to such modifications, which include acetylation, phosphorylation, methylation, and ubiquitination, among others. This type of site-specific histone modification has been implicated in a lot of important processes in eukaryotic cells, such as gene regulation, DNA repair, DNA recombination, and chromatin structure remodeling [2]. In 2000, Strahl and Allis proposed that distinct histone modifications can act sequentially or in combination to form a "histone code", which can be read by other proteins to bring about distinct downstream events [3]. This epi-

*These authors contributed equally to this work. Correspondence: Xing Wang Deng

Tel: 86-10-8072-6675; Fax: 86-10-8072-7509;

E-mail: xingwang.deng@yale.edu

Received 17 Jan 2006; revised 14 Mar 2006; accepted 21 Mar 2006; published online 15 May 2006 genetic "code" thus considerably extends the information potential of the genetic code [1].

In 1989, the first antiserum for site-specific acetylation of histone $\mathrm{H} 4$ was generated by immunization with synthetic peptides $[4,5]$. Since then, many antibodies for histone sitespecific modifications have been generated and widely used to study histone modification. Although almost 20 years has passed since the first antibody against a histone sitespecific modification was developed, the availability and affordability of such antibodies is still a limiting factor in this area of research [6]. However, commercially produced antibodies are expensive and quality may vary from batch to batch. Thus an inexpensive and reliable source of high affinity antibodies against specific histone modifications is important for this area of research.

Since the development of monoclonal antibody production 30 years ago by Cesar Milstein and Georges Kohler, it is clear that monoclonal preparation is much better than polyclonal serum in achieving high specificity. Owing to the small size of the spleen, traditional methods of preparing mouse or rat monoclonal antibodies are limited in terms of capacity for high throughput screening of highly specific 
antibodies. The larger spleen of rabbits can help to greatly expand the capacity for screening highly specific monoclonal antibodies [7], thus greatly increasing the chances of obtaining optimal antibodies. In 1995, Polet et al. generated a rabbit plasmacytoma cell line that made the generation of rabbit monoclonal antibodies possible [7]. This kind of monoclonal antibody is superior in terms of its specificity, binding affinity, and bioactivity in a wide variety of biological assays. Further, it is believed that rabbits can recognize more antigens and epitopes than mice or rats [7]. Thus the rabbit system is better suited for production of monoclonal antibodies against site-specific histone modifications.

Although histone modification was first discovered more than 40 years ago [8], many fundamental questions about the biology of histone modifications remain unresolved. Previous studies have established that histone modification is enormously complicated. In different organisms, the site [9], sequence [5, 10], and level of modification [2, 11] may be different in some cases, while conserved in others. Although there is vast information available about the functional role of specific histone modifications [12-14], the potential role of histone modification in regulation of development has remained elusive. In this study, we report the characterization of five antibodies that specifically target four modified histone sites and the initial application of those antibodies to determine the levels of those four specific histone modifications in different species as well as in different organs of the selected species.

\section{Materials and methods}

\section{Antibody preparation, specificity, and utility assays}

We synthesized modified peptides corresponding to the histone modification sites in Arabidopsis (peptide sequences are described in Table 1). All of those peptide sequences are conserved among histones found in mammals and higher plants. The rabbit monoclonal antibodies were produced in cooperation with Epitomics Biotech Co. Ltd. (Hangzhou, China), and the rabbit polyclonal antibodies were prepared by the Shanghai Sangon Biotech Company (Shanghai, China). We simultaneously expressed full-length His-tagged forms of recombinant Arabidopsis histones $\mathrm{H} 3$ and $\mathrm{H} 4$ in E.coli. The purified full-length $\mathrm{H} 3$ and $\mathrm{H} 4$ recombinant histone proteins were used as antigens to generate polyclonal antibodies in rabbits specific for unmodified histones $\mathrm{H} 3$ and $\mathrm{H} 4$, respectively. The specificity assays performed included immunoblot analysis, dot-blot analysis, and peptide inhibition analysis. For the immunoblot analysis, acidextracted histone protein samples were separated on $15 \%$ sodium dodecyl sulfate-polyacrylamide gels (SDS-PAGE) and the proteins were transferred to a PVDF membrane. After blocking with freshly prepared PBS containing 5\% non-fat dry milk (PBS-milk) for $1 \mathrm{~h}$ at room temperature (RT) with constant agitation, we incubated the PVDF membrane with primary antibodies diluted in freshly prepared PBS-milk solution for $1 \mathrm{~h}$ at RT or $4^{\circ} \mathrm{C}$ overnight. For $\alpha-\mathrm{H} 3 \mathrm{~K} 4 \mathrm{Me} 2$ (clone YCA-47-1-4), the final concentration of $\operatorname{IgG}$ was 0.1-0.2 $\mu \mathrm{g} / \mathrm{ml}$; for a-H3K9Ac (clone YCA-28-14), the final concentration of
IgG was $0.2-0.5 \mu \mathrm{g} / \mathrm{ml}$; for $\alpha-\mathrm{H} 3 \mathrm{~K} 9 \mathrm{Me} 2$ (clone YCA-49), the final concentration used was $1-1.5 \mu \mathrm{g} / \mathrm{ml}$; for $\alpha-\mathrm{H} 4 \mathrm{~K} 12 \mathrm{Ac}$ (polyclonal antibody), the dilution was 1:4000; and for $\alpha-\mathrm{H} 4 \mathrm{~K} 12$ (polyclonal antibody), the dilution was 1:2000. After incubating the membrane with the secondary antibody (goat anti-rabbit HRP-conjugated IgG, Sigma), we developed the signal using the ECL plus Western blot detection reagents (Amersham) and analysis procedures.

To perform the dot-blot analysis, we spotted $1 \mu 1$ of peptide diluted in sterile water to $50,10,2$, and $0.4 \mathrm{pmol} / \mu$ l onto the PVDF membrane and allowed it to air dry. The PVDF membrane was then blocked in a manner similar to that used in the immunoblot analysis. For the peptide inhibition analysis, about $2 \mathrm{mg}$ of the acid-extracted Hela cell histone samples were loaded on an SDS-PAGE mini gel in 48 identical lanes and transferred to a PVDF membrane. After blocking, we cut the membrane into strips of equal size (with one lane each). We added each of the indicated inhibitor peptides to the diluted primary antibodies (the final peptide concentrations were 5 $\mu \mathrm{M}$ for $\alpha-\mathrm{H} 3 \mathrm{~K} 4 \mathrm{Me} 2, \alpha-\mathrm{H} 3 \mathrm{~K} 9 \mathrm{Ac}$, and $\alpha-\mathrm{H} 3 \mathrm{~K} 9 \mathrm{Me} 2$, and $10 \mu \mathrm{M}$ for $\alpha-\mathrm{H} 4 \mathrm{~K} 12 \mathrm{Ac}$ ) and incubated at RT for $1 \mathrm{~h}$ before incubating the membrane strips with the indicated primary antibodies. The procedure following primary antibody incubation is the same as for immunoblot analysis. Chromatin immunoprecipitation (ChIP) assays were performed as described previously [15]. For $\alpha-\mathrm{H} 3 \mathrm{~K} 4 \mathrm{Me} 2$, the amount of antibody is $2.5 \mu \mathrm{g} / \mathrm{ml}$; for $\alpha-\mathrm{H} 3 \mathrm{~K} 9 \mathrm{Ac}$, it is $3 \mu \mathrm{g} / \mathrm{ml}$; and for $\alpha-\mathrm{H} 4 \mathrm{~K} 12 \mathrm{Ac}$ (polyclonal antibody), it is $5 \mu \mathrm{serum} / \mathrm{ml}$.

\section{Materials and histone extraction}

Six organs (flowering bud, silique, stem, root, rosette leaf, and cauline leaf) from our plant model, wild-type Arabidopsis thaliana (Col.), were selected for organ-specific analysis of histone modification levels. For our mammalian model, the heart, liver, spleen, lung, and kidney of adult mouse were used in a similar analysis. Other representative species and organ types used included rice leaf, human-cultured cells (Hela), and Calf thymus. The histones from plant materials were isolated as described previously [11]. Acid extractions of histones from Hela cells were performed on cell cultures grown at $70-90 \%$ confluency in $1 \times$ Dulbecco's-Modified Eagle's Medium (high glucose) supplemented with $10 \%$ FBS. To obtain a high acetylation levels in our histone sample, we added sodium butyrate (SB), which inhibits histone deacetylases, to a final concentration of 5 $\mathrm{mM}$, which was maintained for $24 \mathrm{~h}$ before harvesting the cells. We scraped the cells from the plate, and after pelleting and washing the cells, the cell pellet was suspended in 5-10 volumes of lysis buffer (10 mM HEPES, pH 7.9, $1.5 \mathrm{mM} \mathrm{MgCl}_{2}, 10 \mathrm{mM} \mathrm{KCl}, 0.5 \mathrm{mM}$ DTT, $1.5 \mathrm{mM}$ PMSF). We added $\mathrm{H}_{2} \mathrm{SO}_{4}$ to a final concentration of $0.4 \mathrm{M}$ and incubated the suspension on ice for at least $4 \mathrm{~h}$. After spinning down to pellet the cell debris, $1 / 3$ volume of $100 \%$ TCA was added to the supernatant and the mixture was incubated on ice for $30 \mathrm{~min}$. The histone proteins were pelleted by centrifugation and were washed with acetone. The extracted histone protein pellet was suspended with $2 \times$ Loading Buffer and the $\mathrm{pH}$ was adjusted with $\mathrm{NaOH}$ until the color of the loading buffer turned blue. The animal tissues were homogenized in cold PBS plus $1 \mathrm{mM}$ freshly added PMSF using a glass-Telfon homogenizer. The homogenate was filtered through a layer of Miracloth, the filtrate was spun down, and the pellets were processed using procedures identical to those for the Hela cells.

\section{Immunoblot analysis}

Acid-extracted proteins were separated by electrophoresis in $15 \%$ 
or $18 \%$ SDS-PAGE and transferred to PVDF (Millipore) membrane in Tris-glycine buffer. The membrane was stained by Ponceau $\mathrm{S}$ or Fast Green. After washing in PBS-T to remove the dye, the membrane was blocked by PBS containing 3\% non-fat dry milk for $30 \mathrm{~min}$ and then probed with indicated histone antibody as the primary antibody at the same dilution with antibodies assaying. The secondary antibody was anti-rabbit IgG at a dilution of 1:8,000 (Sigma). We repeated each experiment at least twice with both our own and the Upstate antibodies to confirm the reproducibility.

\section{Results}

\section{Antibody specificity}

Previous studies have established that acetylation of histones $\mathrm{H} 3 \mathrm{~K} 9$ and $\mathrm{H} 4 \mathrm{~K} 12$ and methylation of histone H3K4 are conserved markers for gene activation and euchromatin formation, while methylation of histone $\mathrm{H} 3 \mathrm{~K} 9$ is a marker for gene silencing and heterochromatin formation [1, 11, 16]. These four site-specific modifications with known biological function were selected for the production of rabbit monoclonal and polyclonal antibodies. The specific peptides used as antigens are summarized in Table 1. To test antibody specificity, we first tested our site-specific antibodies against recombinant histone proteins and the acid-extracted histone samples treated with $+\mathrm{SB}$ or untreated -SB Hela cells. It is anticipated that SB treatment will inhibit histone deacetylation and result in high levels of acetylated histone accumulation. As shown in Figure 1, our site-specific antibodies against $\mathrm{H} 3 \mathrm{~K} 9 \mathrm{Ac}$ and H4K12Ac both detected strong bands in SB-treated samples while

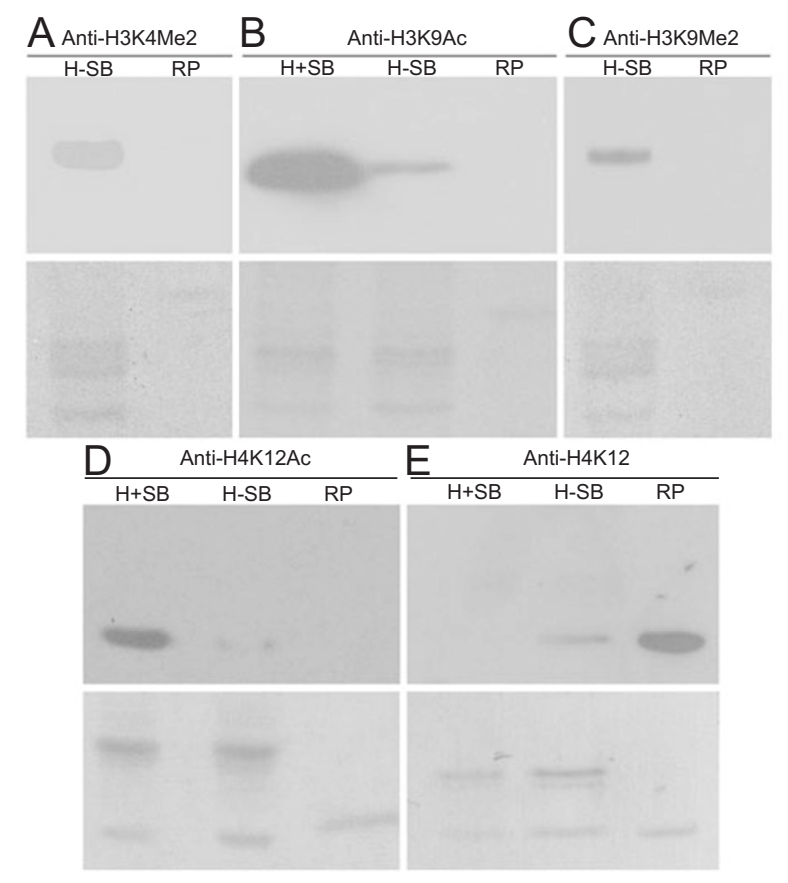

Figure 1 Antibody specificity tested by immunoblot analysis. The top panels show the immunoblots with the five custom-made antibodies for site-specific modified histones against acid-extracted proteins from Hela cells treated with $5 \mathrm{mM} \mathrm{SB}$ for $24 \mathrm{~h}(\mathrm{H}+\mathrm{SB})$, normal Hela cells (H-SB), and recombinant histones (RP). The bottom panels show the membrane or the SDS-PAGE gel used for the immunoblot to confirm equally loading. Note: the recombinant histone $\mathrm{H} 3$ has a His-tag at its $\mathrm{N}$ terminus, so it looks bigger than native protein. Recombinant histone $\mathrm{H} 4$ was purchased from the Upstate Company. $\mathrm{SB}$ is an inhibitor of histone deacetylase.

Table 1 Summary of our antibodies and their tested applications

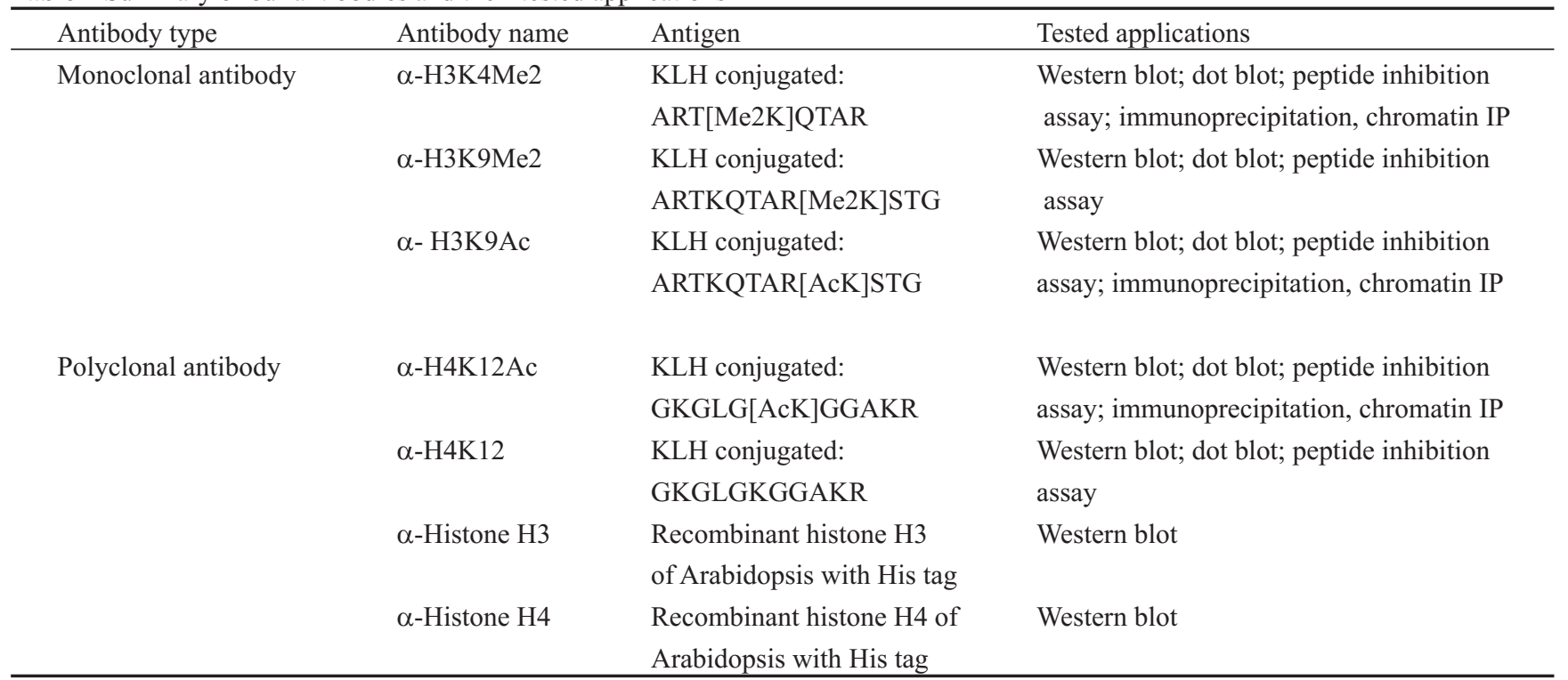

Abbreviation: Me2K, lysine with dimethylation; Ac, acetylation. 
the band was weak or absent in untreated samples. Those same antibodies do not react at all with recombinant histone proteins loaded at an amount equivalent to that of the extracted histones. The antibodies against $\mathrm{H} 3 \mathrm{~K} 4 \mathrm{Me} 2$ and $\mathrm{H} 3 \mathrm{~K} 9 \mathrm{Me} 2$ can only recognize histones from Hela cells, which presumably contain a certain level of those site-specific modifications, but these antibodies do not recognize recombinant histone $\mathrm{H} 3$ proteins at all. In a control, antibodies against the unmodified H4K12 specifically recognized the recombinant histone protein and reacted weakly to Hela cell extracts untreated with SB, which are expected to have low levels of unmodified histone $\mathrm{H} 4$ at the K12 site. However, this antibody hardly reacts with histone H4 extracted from SB-treated Hela cells, possibly owing to higher acetylation and lower unmodified level at the K12 site.

We also used dot-blot analysis to test the specificity of our antibodies. As shown in the top panels of Figure 2, all five site-specific antibodies, four modified forms and one unmodified form, only reacted with the specific peptide and not with any other peptides tested, in a range of peptide concentrations from a sub-picomolar amount to $50 \mathrm{pmol}$. This result suggested that our antibodies possess the desired specificity at the substrate concentration range normally used in routine experimentation.

We further tested our histone modification site-specific antibodies for possible crossreactivity using a peptide inhibition assay (bottom panels of Figure 2). Again, most of our antibodies can only be inhibited by the specific target peptides, not by other peptides. Only in the case of our antibody for H3K9Me2, a weak inhibition by the H3K4Me2 peptide and a moderate inhibition by the H3K27Me2 peptide (Table 1) were observed. This partial inhibition was likely due to common amino acids present in the peptides. Both the H3K27Me2 and H3K9Me2 shared an "AR[Me2 K]S" amino acid motif, which might be part of the epitope

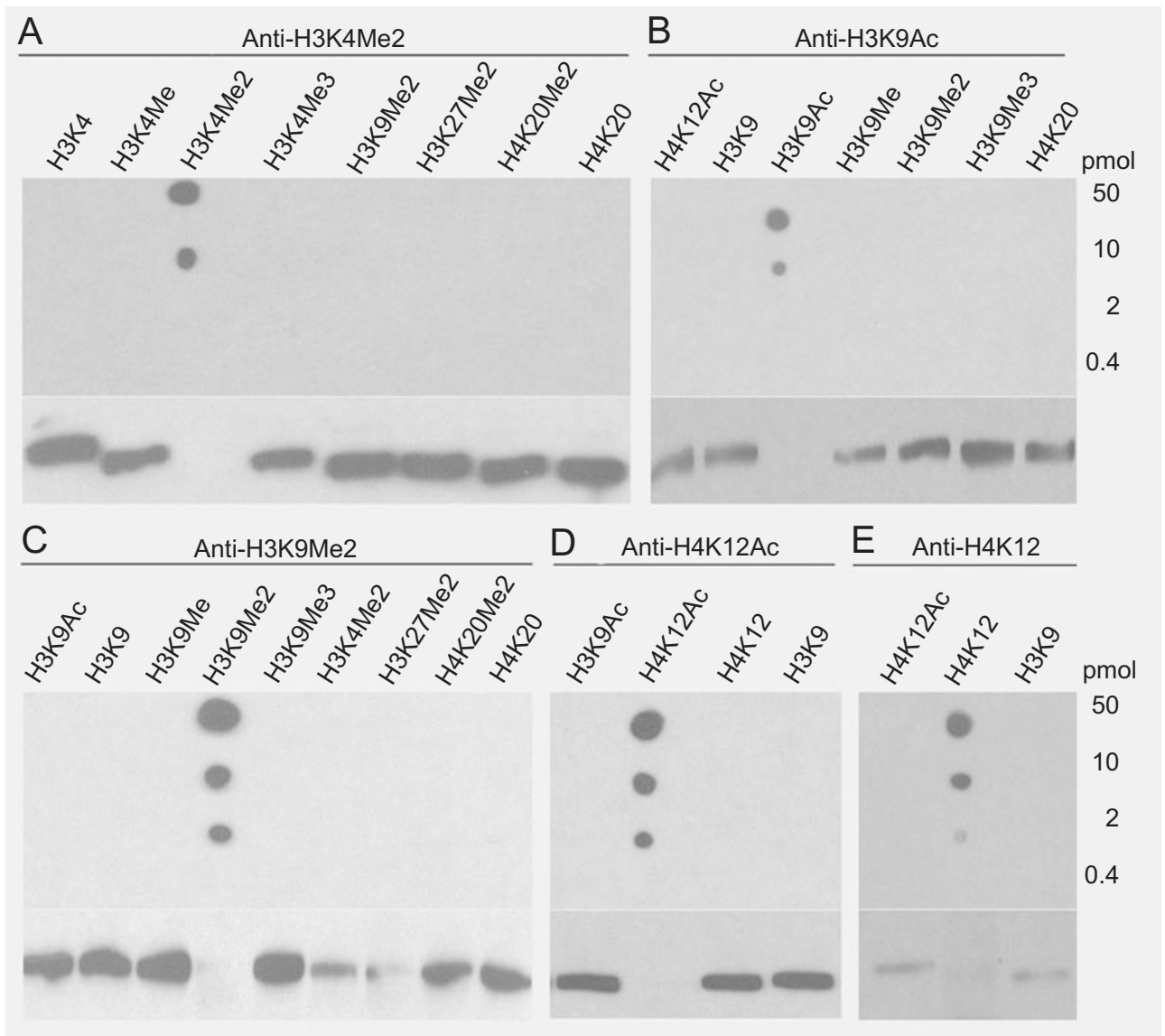

Figure 2 Dot-blot (top panels) and peptide inhibition analyses (bottom panels) test cross reactivity of antibodies. The amounts of the corresponding peptides used for dot-blot analysis are indicated at the right. In the peptide inhibition assay, the indicated peptides are added to diluted antibodies before being used for immunoblot analysis with acid-extracted proteins from Hela cells untreated with SB. 


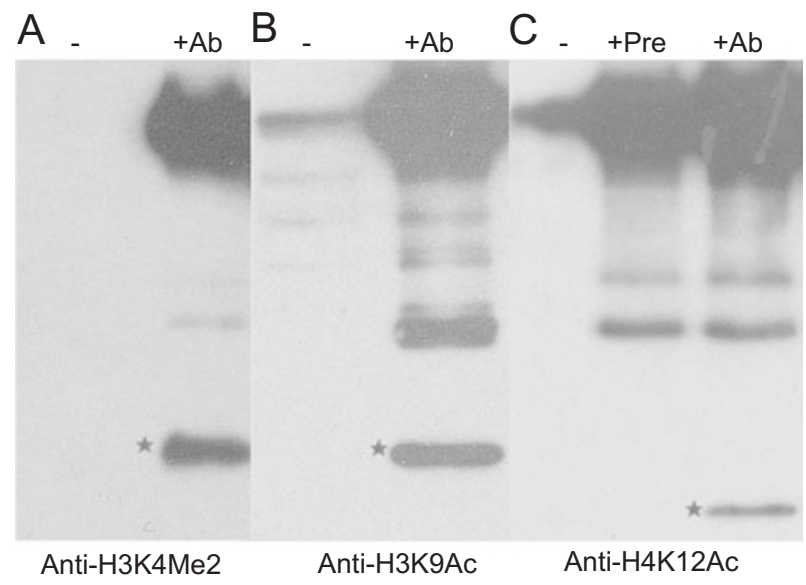

Figure 3 Three site-specific histone modification antibodies are suitable for immunoprecipitation analysis. Each of the three antibodies $(+\mathrm{Ab})$ for H3K4Me2 (A), H3K9Ac (B), and H4K12Ac (C) was used to immunoprecipitate histone samples. A control (-) without antibody for all the three antibodies and a pre-immune serum (+Pre) control used for polyclonal antibodies were included. The immunoprecipitates were separated on $15 \%$ SDS-PAGE and blotted with antibodies purchased from Upstate corresponding to the same histone modification sites.

recognized by this antibody for $\mathrm{H} 3 \mathrm{~K} 9 \mathrm{Me} 2$. The $\mathrm{H} 3 \mathrm{~K} 4 \mathrm{Me} 2$ peptide has an amino acid motif "ART[Me2K]", similar but not identical to that of H3K9Me2 peptide, which could explain its weak inhibition effect. Since histone H3K9 shares a similar flanking amino acid sequence to $\mathrm{H} 3 \mathrm{~K} 4$ and $\mathrm{K} 27$, it is a possibility that the antibodies can crossreact to $\mathrm{H} 3 \mathrm{~K} 4$ and $\mathrm{K} 27$ [17]. A lot of antibodies commercially available for $\mathrm{H} 3 \mathrm{~K} 9 \mathrm{Me} 2$ also seemed to have more or less some degree of crossreactivity, including widely used antibodies from Upstate Company (for example, product \#05-768). Using a different immunization strategy such as immunizing with two-branched peptides, it was claimed that the resulting antibodies (Upstate \#07-441) for H3K9Me2 had minimal crossreaction with $\mathrm{H} 3 \mathrm{~K} 4 \mathrm{Me} 2$ or $\mathrm{H} 3 \mathrm{~K} 27 \mathrm{Me} 2$ $[17,18]$. However, this strategy of not immunizing with original amino-acid sequences might produce antibodies that crossreact with other structural features in the organism or variant histone proteins.

\section{Assessment of antibody suitability for immunoprecipitation and ChIP analyses}

The antibodies for detecting site-specific modifications of histones can be powerful tools for analysis of histone modification in vivo using immunoblot, immunoprecipitation (IP), and ChIP assays. Our specificity analyses shown in Figures 1 and 2 demonstrated our antibodies' utility for immunoblot and dot-blot analyses. We then further exam-
A

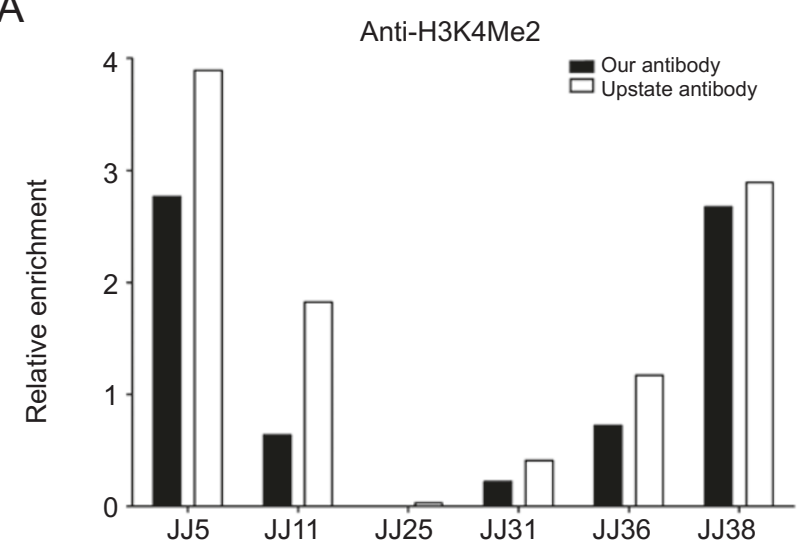

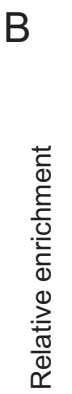
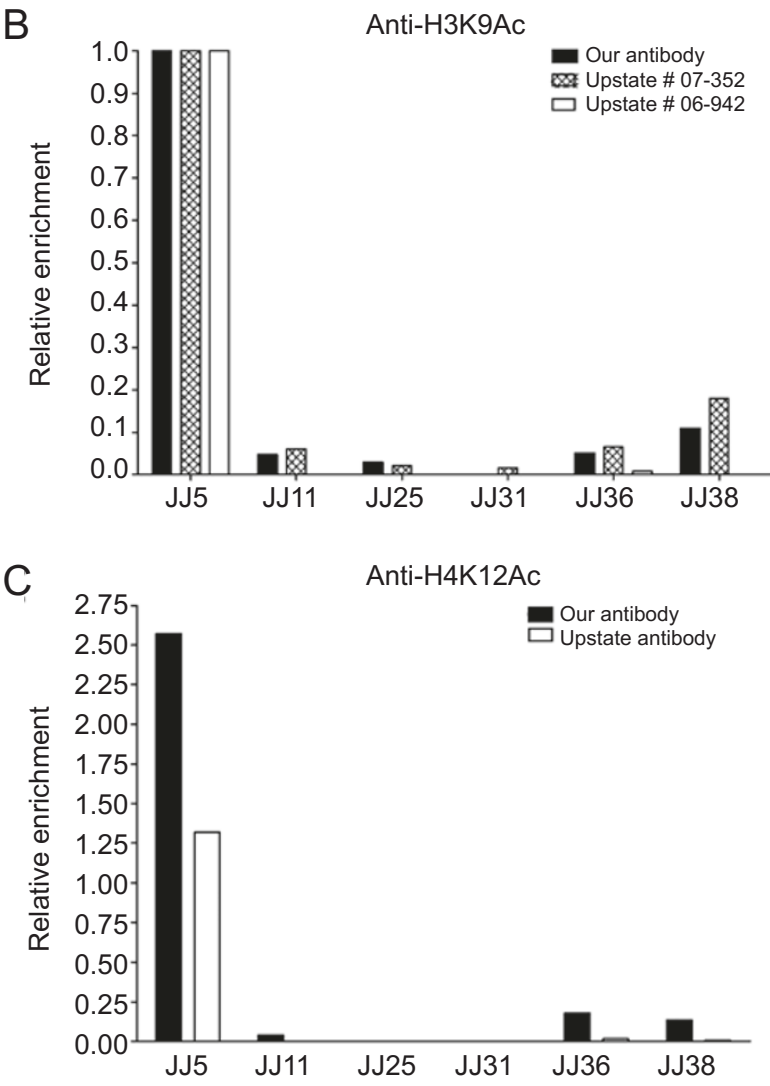

Figure $4 \mathrm{ChIP}$ analysis of custom-made antibodies compared to purchased antibodies. The three diagrams (A-C) display the relative enrichment ( $Y$-axis) of corresponding histone modifications at specific sites, as indicated by the primer numbers described previously [19]. All selected primers sites correspond to the regions in the rice chromosome 8 centromere. The normalization formula is as follows: relative enrichment $=\left(\mathrm{ChIP}_{\text {antibody }}-\mathrm{Mock}\right) /$ Input. The results using our antibodies and Upstate antibodies are shown side by side.

ined our antibodies' utility in IP and ChIP assays, since it is known that some antibodies can be used for one assay but 
not for the others [6]. Among the five specific antibodies we have tested, three antibodies (for H3K4Me2, H3K9Ac, and $\mathrm{H} 4 \mathrm{~K} 12 \mathrm{Ac}$ ) are able to specifically precipitate their cognate modified histones, as confirmed by immunoblot with specific antibodies against the modified site (Figure 3).

To test antibody specificity and suitability for ChIP assays, we compared the performance of our three site-specific antibodies suitable for IP side-by-side with commercial antibodies from the Upstate Company in a ChIP assay for modified histones associated with several known rice genome sites. We selected six primer pairs corresponding to the sequences near the centromere of rice chromosome 8 reported previously [19] for the ChIP assay. As shown in Figure 4, all three of our site-specific antibodies essentially displayed the same histone modification patterns as the antibodies purchased from the Upstate Company. We also tested the ChIP ability of our antibodies with Arabidopsis and compared it to the ChIP ability of the Upstate antibodies. Again, no significant differences were seen (data not shown).

Levels of all four site-specific histone modifications were quantitatively similar in different organs of Arabidopsis and mouse

It is widely recognized that histone modification is a dynamic process. Under different conditions, histone modification could be reversibly regulated in some specific regions of the genome [20-22]. However, there is currently no report of overall changes in site-specific histone modification levels in different organs of the same organism.
We thus used our antibodies to test the overall levels of four site-specific histone modifications in different organs of Arabidopsis and mouse. As shown in Figure 5, no significant differences in any of the levels of the site-specific modifications were found among distinct organs of either Arabidopsis or mouse. This suggests that overall levels of site-specific histone modification do not show significant differences among different organs in a given organism. We also tested modification levels by immunoblot analysis using corresponding antibodies from Upstate, and obtained resutlts identical to those shown for our antibodies (data not shown).

Overall levels of site-specific modifications are highly variable among distinct organisms

Although histones are characterized by highly conserved amino-acid sequences, potential differences may exist in the specific characteristics and extent of site-specific histone modifications. To examine possible differences in site-specific histone modifications among several species, we analyzed the overall levels of four site-specific histone modifications with our antibodies. As shown in Figure 6 , there are some clear differences in the extent of some site-specific modifications. For example, in Arabidopsis Columbia ecotype, there was a low level of histone H3K9 dimethylation modification, but other modifications were very high comparing with other species. It has been reported that histone $\mathrm{H} 3 \mathrm{~K} 4$ dimethylation, $\mathrm{H} 3 \mathrm{~K} 9$, and $\mathrm{H} 4 \mathrm{~K} 12$ acetylation are correlated positively [1]; whereas all of those three site-specific modifications have an inverse cor-

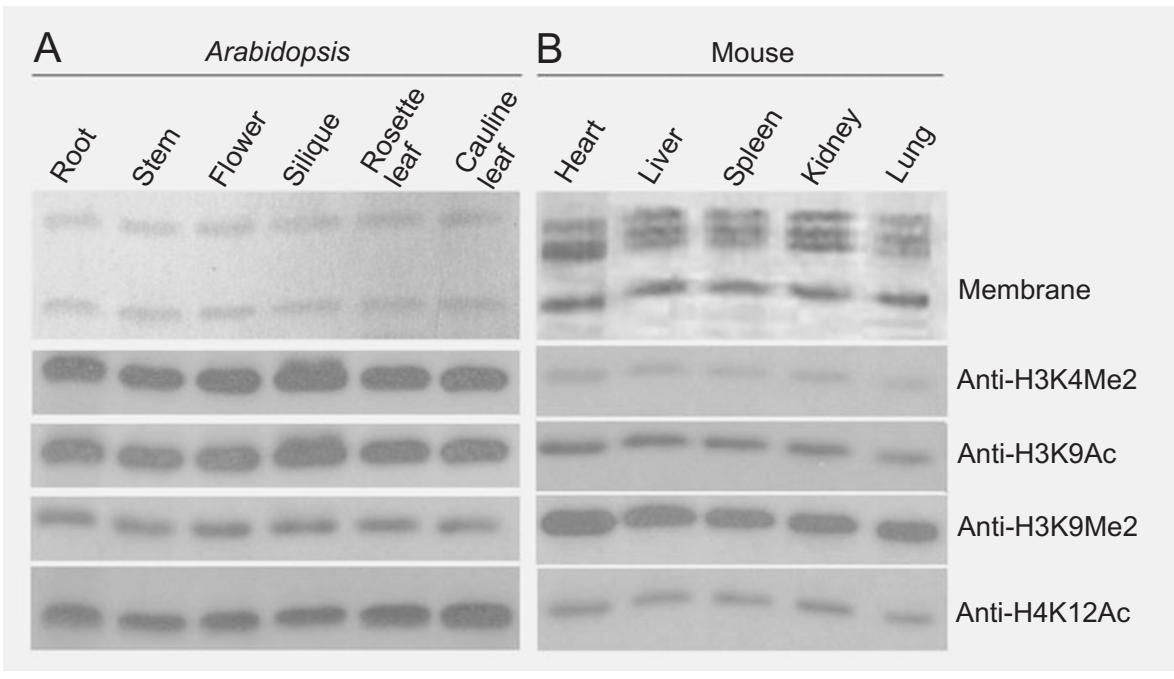

Figure 5 Western blot analysis of overall site-specific histone modification levels among different organs of Arabidopsis (A) and mouse (B). The top panels are the stained membranes used for immunoblot analysis, which show the equal loading of the histone proteins. The panels below are the results of immunoblot analysis with the corresponding antibodies indicated at the right. 


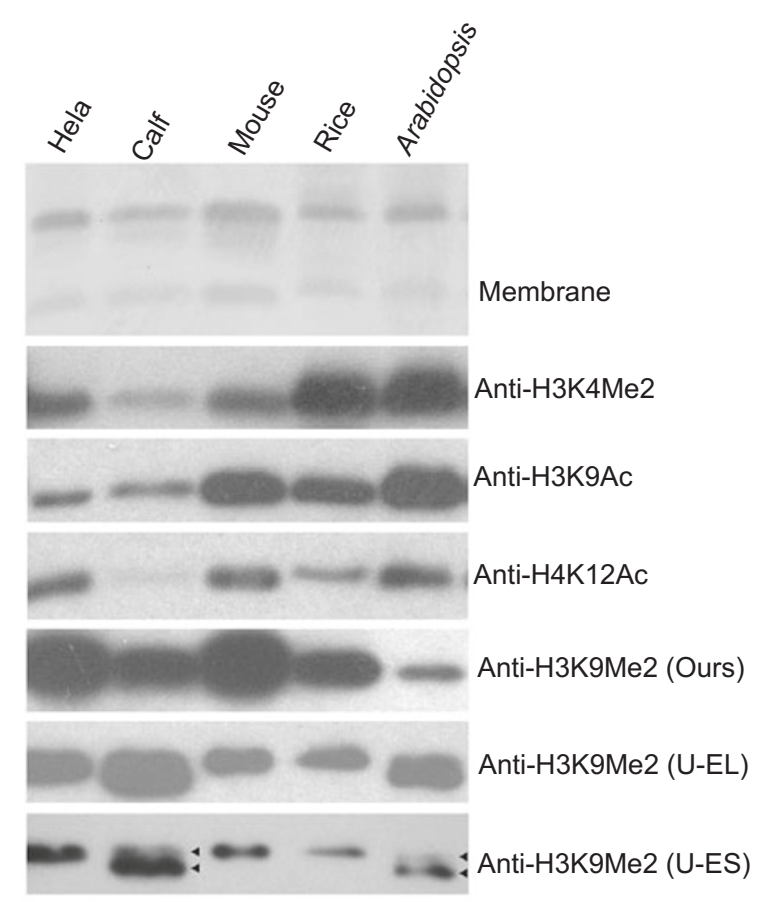

Figure 6 Immunoblot analysis of overall site-specific histone modification levels among different organisms. The top panel shows a representative stained membrane used for immunoblot analysis, to show equal loading of the histone proteins from different organisms. The panels below show the immunoblot results with the corresponding antibodies indicated at the right. We ran an 18\% SDS-PAGE gel to assess the efficacy of anti-H3K9Me2. The last two panels were from the same Western blot analysis with the Upstate antibodies (\#07-441), but with a longer (U-EL) or shorter (U-ES) exposure time. The two bands (as marked by the triangles) in calf and Arabidopsis Columbia ecotype were clearly visible in the shorter exposure Western blot.

relation with histone $\mathrm{H} 3 \mathrm{~K} 9$ dimethylation [1]. This general relationship is also evident in the number of total modifications as shown in Figure 6. Repetition of immunoblot analyses using the corresponding antibodies purchased from Upstate yield very similar results (data not shown), with the exception of H3K9Me2. For the Upstate antibodies for H3K9Me2 (\#07-441), the antibodies also react with an additional band slightly below the expected specific band in Calf and Arabidopsis Columbia ecotype samples. This was especially evident in the immunoblot with shorter exposure time (Figure 6). In both calf and Arabidopsis samples, comparison of only the upper specific bands for the Upstate antibody and our own antibody suggests that the two antibodies perform equally well.

\section{Discussion}

Antibodies that can distinguish between modified and unmodified histones or among different modified forms constitute an essential tool for studying the functional role of site-specific histone modifications. Since the development of the first antibody for a modified histone, there has been a great demand for reliable and highly specific antibodies for various site-specific histone modifications. However, most antibodies available in the past are polyclonal antisera, which are always limited in quantity and the quality is often highly variable from batch to batch. Thus obtaining monoclonal hybrid myeloma cells that are capable of producing unlimited amounts of high-quality monoclonal antibodies for each possible site-specific histone modification is an important goal. Since hybrid myeloma cells can be propagated indefinitely, they provide an inexhaustible source for the same high-quality monoclonal antibodies for a given site-specific histone modification, while also ensuring high reproducibility. Our work demonstrates the feasibility of raising a sizable quantity of polyclonal antibody sera from rabbits, while simultaneously preparing monoclonal antibodies using the spleen of the same rabbit. Using this approach, we have generated antibodies (three monoclonal and one polyclonal) for detecting site-specific histone modifications.

We compared our custom-made antibodies with antibodies against the same site-specific modified histones from a common commercial source (Upstate Company) to assess our antibodies' performance in ChIP and immunoblot analyses. For antibodies against H3K4Me2, three different products from Upstate were compared: the first is a polyclonal rabbit serum (\#07-030); the second is rabbit monoclonal IgG (clone AW30; \#05-790); and the third is mouse monoclonal IgM (clone RR302; \#05-684). Of the three, only rabbit serum can be used in ChIP experiments, whereas both monoclonal antibodies of mouse and rabbit origin cannot. Our monoclonal $\operatorname{IgG}$ antibody can be used in ChIP experiments, although the signal is slightly weaker compared to the signal generated using Upstate's rabbit polyclonal serum (as shown in Figure 4A). For antibodies against H3K9Ac, the Upstate Company has two products. One of them is protein A purified rabbit IgG (\#06-942) and the another is whole rabbit antiserum (\#07-352), both of which can be used to do ChIP experiments. However, the purified rabbit IgG (\#06-942) gave a weak signal in the ChIP experiment, while the rabbit antiserum (\#07-352) crossreacted to a non-specific band positioned slightly higher than the histone H3 band that can easily be confused with certain site-modified histone $\mathrm{H} 3$ forms (data not shown). Our monoclonal IgG antibody for H3K9Ac performs well in ChIP experiments and the signal is superior to that of the purified rabbit IgG antibody from the Upstate Company. For antibodies against H3K9Me2, the Upstate Company offers six products, only one (\#07-441) of which 
can be used in ChIP experiments. Perhaps due to use of a different immunization strategy, this antibody can detect an additional crossreacting band in calf and Arabidopsis Columbia ecotype that was very close to the specific band (Figure 6, the last 2 panels). Although our antibody against $\mathrm{H} 3 \mathrm{~K} 9 \mathrm{Me} 2$ is not suitable for ChIP experiments, it performs very well in immunoblot analysis. For antibodies against H4K12Ac, the Upstate Company offers two products. One of them is protein A purified rabbit IgG, and the other is the rabbit antiserum depleted of non-acetyl reactive antibodies. Only the latter can be used in ChIP experiments. In a side-by-side comparison of the ChIP results (Figure 4C), our polyclonal antibody against H4K12Ac shows a much stronger signal compared with that of the Upstate antibodies. In immunoblot experiments, our antibody also yields a better signal even at high dilutions (data not shown), implying the very high affinity of our antibodies. Taken together, these results suggest that our antibodies exhibit many properties comparable or superior to commercially available ones.

Owing to their central function in all eukaryotic organisms, histones are a highly conserved group of proteins. The sequence of the most highly conserved group member, histone H4, differs in Arabidopsis and mammals by only two amino acids, although plants and animals diverged about 1.6 billion years ago. However, Arabidopsis and mammals display significant differences in histone modification. It was thought that as plant cells have retained the property of totipotency, or ability to regenerate the entire plant from a single cell, and maintained the ability to de-differentiate, the establishment and maintenance of epigenetic information might differ significantly from animals [9]. Some species might display special histone modification patterns [2] and modification sites (http://research.nhgri.nih.gov/histones/index.shtml) that were not observed in other species [2]. The same modification might function differently in unrelated species $[1,2,11,14,16]$. Previous studies also showed that some site-specific histone modification differences between animals and plants exist at a genome-wide level [11]. Thus, it is of great interest to know if the extent of site-specific modifications varies among different organs of a given species and among different species.

Our studies revealed that there is no overall difference in the extent of site-specific modifications among different organs of a given organism. Thus, the overall role of histone modification in the regulation of an organism's development appears to be limited. The level of a site-specific histone modification of any organ can be an indicator for the level in the whole organism. However, this observation clearly does not rule out potential differences among localized and specific regions of a chromosome during the development of an organism $[2,17,18]$.
However, there is a great variation in the overall level of site-specific histone modifications among distinct organisms. This may reflect the different developmental patterns and life cycles of different organisms [9]. It is interesting to note that our data on the overall extent of histone modification among organisms examined further substantiated the previously observed positive correlation among histone modifications (H3K4Me2, H3K9Ac, H4K12Ac) associated with gene activation. Our data also corroborated an inverse correlation among histone modifications (H3K9Me2) associated with gene repression at the local chromatin and gene level. These findings further suggest roles for these site-specific histone modifications in modulating gene activity. The availability of this set of site-specific histone modification antibodies will provide essential tools for further examining the functional implications of histone modification in various organisms and developmental processes.

\section{Acknowledgements}

We thank Shunong Bai for providing Calf histone protein sample, Yuling Jiao, Ligeng Ma, and Jiwan Qiu for helpful discussion and suggestions in antibody production, Ning $\mathrm{Cai}$, Ting $\mathrm{Fu}$, and Taotao Lao of the Chinese Division of Epitomics Biotech Co. Ltd. for production of monoclonal antibodies, and Valerie $\mathrm{J}$ Karplus for critical reading of this manuscript. This research was funded by the Hi-Tech Research and Development Program of China (Grant 2003-AA210070).

\section{References}

1 Jenuwein T, Allis CD. Translating the histone code. Science 2001; 293: 1074-1079.

2 Jasencakova Z, Soppe WJJ, Meister A, et al. Histone modifications in Arabidopsis-high methylation of H3 lysine 9 is dispensable for constitutive heterochromatin. Plant J 2003; 33:471-480.

3 Strahl BD, Allis CD. The language of covalent histone modifications. Nature 2000; 403:41-45.

4 Turner BM, Fellows G. Specific antibodies reveal ordered and cell-cycle-related use of histone-H4 acetylation sites in mammalian cells. Eur J Biochem 1989; 179:131-139.

5 Turner BM, Laura PO, Allan IM. Histone H4 acetylation in human cells frequency of acetylation at different sites defined by immunolabeling with site-specific antibodies. FEBS Lett 1989; 253:141-145.

6 Suka N, Suka Y, Carmen AA, Wu J, Grunstein M. Highly specific antibodies determine hisotne acetylation site usage in yeast heterochromatin and euchromatin. Mol Cell 2001; 8:473-479

7 Polet HS, Sethupathi P, Yam P-C, Knight KL. Rabbit monoclonal antibodies: generating a fusion partner to produce rabbit-rabbit hybridomas. Proc Nat Acad Sci. USA 1995; 92:9348-9352

8 Allfrey VG, Faulkner R, Mirsky AE. Acetylation and methyla- 
tion of histones and their possible role in the regulation of RNA synthesis. Proc Nat Acad Sci USA 1964; 51:789-794

9 Loidl P. A plant dialect of the histone language. Trends Plant Sci 2004; 9:84-90

10 Couppez M, Annie MP, Sautiere P. Histone H4 from Cuttlefish Testis is sequentially acetylated. J Biol Chem 1987; 262:28542860

11 Jackson JP, Johnson L, Jasencakova Z, et al. Dimethylation of histone H3 lysine 9 is a critical mark for DNA methylation and gene silencing in Arabidopsis thaliana. Chromosoma 2004; 112:308-315

12 Ringrose L, Ehret H, Paro R. Distinct Contributions of Histone H3 Lysine 9 and 27 Methylation to Locus-Specific Stability of Polycomb Complexes. Mol Cell 2004; 16:641-653.

13 Bastow R, Mylne JS, Lister C, et al. Vernalization requires epigenetic silencing of FLC by hisotne methylation. Nature 2004; 427:164-167.

14 Briggs SD, Bryk M, Strahl BD, et al. Histone H3 lysine 4 methylation is mediated by Set1 and required for cell growth and rDNA silencing in Saccharomyces cerevisiae. Genes Dev 2001; 15:3286-295.

15 Gendrel AV, Lippman Z, Martienssen R, Colot V. Profiling histone modification patterns in plants using genomic tiling microarrays. Nat Methods 2005; 2:213-218
16 Strahl BD, Ohba R, Cook RG, Allis CD. Methylation of hisotne $\mathrm{H} 3$ at lysine 4 is highly conserved and correlates with transcriptionally active nuclei in Tetrahymena. Proc Nat. Acad Sci USA 1999; 96:14967-14972

17 Rice JC, Briggs SD, Ueberheide B, et al. Histone methyltransferases direct different degrees of methylation to define distinct chromatin domains. Mol Cell 2003; 12:1591-1598.

18 Peters AH, Kubicek S, Mechtler K, et al. Partitioning and plasticity of repressive histone methylation states in mammalian chromatin. Mol Cell 2003; 12:1577-1589.

19 Nagaki K, Cheng Z, Ouyang S, et al. Sequencing of a rice centromere uncovers active genes. Nat Genet 2004; 36:138-145.

20 Waterborg JH. Dynamics of histone acetylation in Saccharomyces cerevisiae. Biochemistry 2001; 40:2599-2605.

21 Chua YL, Brown APC, Gray JC. Targeted histone acetylation and altered nuclease accessibility over short regions of the pea plastocyanin gene. Plant Cell 2001; 13:599-612.

22 Chua YL, Watson LA, Gray JC. The transcriptional enhancer of the pea plastocyanin gene associates with the nuclear matrix and regulates gene expression through histone acetylation. Plant Cell 2003; 15:1468-1479. 